Session F3E

\title{
VIRTUAL CLASSROOM FOR MULTIMEDIA TEACHING ON WWW
}

\author{
Iva Bogdanova ${ }^{1}$, Pierre Vandergheynst ${ }^{1}$ and Murat Kunt ${ }^{1}$
}

\begin{abstract}
The specific issue addressed in this paper is tailoring specific functionality to allow a group of teachers and students to carry out the learning process in Digital Signal and Image Processing in an electronic virtual environment that is meant to replace the physical class environment. In order such an environment to be performed, not only the course content itself but also different Webbased tools for interaction between the students and teachers are needed. A Graphical User Interface (GUI), designed and presented here, brings together the students and teachers through virtual class and lab-sessions help and chat rooms. As Digital Signal and Image Processing are areas that heavily depend on mathematics, the lab-sessions are performed through MATLAB Web Server. Some specific applications are shown as well as complementary tools like Web-calendar and Digital Library. Finally, benefits and future work on this virtual classroom are discussed.
\end{abstract}

Index Terms - e-learning, Digital Signal and Image Processing, distance education, multimedia teaching and virtual classroom

\section{INTRODUCTION}

The World Wide Web and other Internet-based collaborative tools have significantly enhanced the ability to train and educate electronically. The Web, when it is integrated with tools such as on-line labs, help-rooms, etc, can increase students' level of involvement in the teaching process. It provides an effective mechanism for integrating many of these tools into a single interface and is an ideal tool for information which is itself rapidly changing. The Web is revolutionizing some areas of study through increased opportunities for learning and alternative formats for information.

A web course, having a teacher and registered group of students, brings together a community of learners into a virtual classroom where they can interact with each other.

The goal is to create a virtual classroom, using methods that will fully engage the students in the learning process through an interactive, dynamic environment involving the student, on-line materials and teacher.

\section{IMPLEMENTATION OF A VIRTUAL ClASSROOM}

\section{Planning of a Virtual Classroom}

The virtual classroom is planned to be in charge of students in engineering and most particularly for courses on Digital Signal and Image Processing. The courses are meant to be wholly on-line and only for students enrolled in the particular course. Finally, the courses' testing and examinations are planned to be wholly online as well.

While testing, it could be a supplement to a class that meets in a traditional classroom, and more then only students inscribed for a course could be able to use it.

\section{Designing of GUI, which serves the Virtual Classroom}

The GUI for the system performing multimedia teaching on WWW is designed to be on four levels (Figure 1). In this way all the capabilities of the system are permanently accessible. On the first level is the Main Interactive Hall, on the second level-Courses, on the third -Course Content, and on the fourth-Support Center.

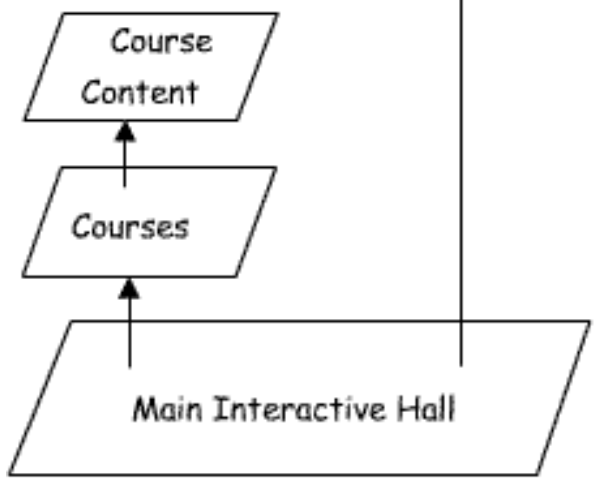

FIGURE. 1

LEVELS IN GUI

The third level is accessible through the second one, meaning the course material can be reached through passing

\footnotetext{
${ }^{1}$ Signal Processing Institute (ITS), School of Engineering (STI), Swiss Federal Institute of Technology (EPFL), CH-1015 Lausanne, Switzerland \{iva.bogdanova; pierre.vandergheynst; murat.kunt\}@epfl.ch

0-7803-7444-4/02/\$17.00 @ 2002 IEEE

November 6 - 9, 2002, Boston, MA

$3^{\text {nd }}$ ASEE/IEEE Frontiers in Education Conference 
descriptions and requirements for taking the course. Support center is directly accessible from the Main Interactive Hall, which is on the first level and consists of three nodes. These are Administration, Support Center and Courses. Organization of the Main Interactive Hall is graphically represented on Figure 2.

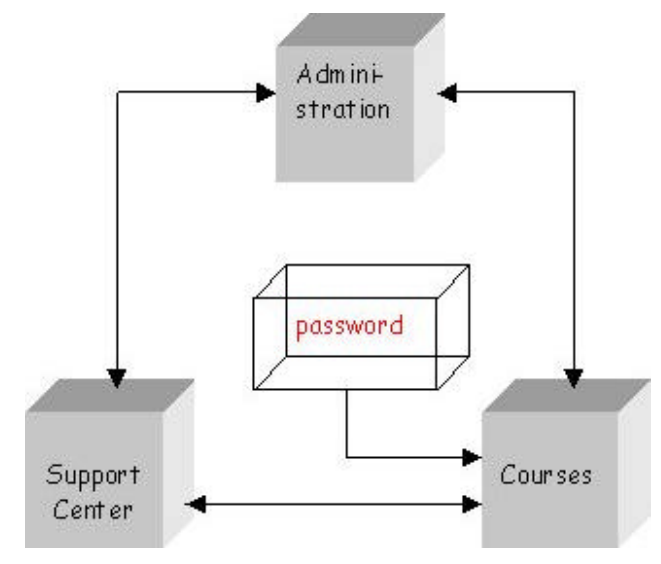

FIGURE. 2

MAIN INTERACTIVE HALL, CONNECTIONS BETWEEN T HE NODES

Administration provides statistics on user and system behavior. Support Center is built from Digital Library, Help room, Chat Room, Students home pages. In Digital Library are available materials that were traditionally on paper or other conventional material, in digital form. Each of the students taking a particular course is presented in Students home pages section. Chat Room is for synchronous communication between students. In Help Room are opened some discussions on course material and this is the place where the students and instructors are meeting. It is a question-answer activity, where if the teacher asks a discussion question, every student must supply an answer before he or she can see the answer of the other students. This is clearly an improvement over the face-to-face class where such discussions are usually dominated by the same small percentage of the students. This feature forces equal participation in any discussion issues the instructor triggers in this manner. It forces each student to do independent thinking about the issue.

Each course is represented by Course Info, Schedule/Calendar, and Announcements; Help Room, Students and Lessons. In Class Info is given basic course information; procedure for taking the course; to students of which semester the course is given; etc. Course schedule is given trough the Schedule/Calendar. Teacher can post the announcements according the course through the Announcements. The Help Room provides whiteboard like a tool for interaction between students and instructor in case of students' questions concerning the course material. The link to the third level, on which is the course material, is through

\section{0-7803-7444-4/02/\$17.00 @ 2002 IEEE}

Lessons. Content is organized in house, rooms, walls and bricks, where the basic concept is represented in a brick. [1] After entering the navigation tool, password verification is required. If the verification is successful, the user is automatically led to the Courses node. From each node are always accessible the other two remaining nodes.

The Administration node is accessible only from users who have administration privilege. Courses and Support Center are accessible from both students and instructors.

\section{Development of GUI}

The development of the GUI is done mainly in Macromedia Flash 5 [2]. Flash excels at being able to deliver graphics for realistic-looking user interfaces. It is a completely vectorbased environment for creating animations. Most of the other methods for creating animations are based on raster images, meaning that they take significantly longer to download with no particular method overcoming the problem of file size.

One of the most interesting features about Flash is its ability to integrate sound. Sound is a very important in creating a system performing multimedia teaching on WWW, since a big amount of information in our case, is carried by it. Sound files that are used within authored Flash movies are stored within the SWF file format. Inside the Flash file, sounds can be compressed with MP3, making it possible to create longplaying animations with voice-overs with smaller files. Moreover, since the audio is stored inside the SWF file, there are no external references or additional HTML code needed.

The Main Interactive Hall is developed in 3D. Interaction for every one of the tree nodes in the GUI consists of buttons. The main button on every one of these stages is an animated 3D cube, through which the user enters into each of the nodes. The other two buttons leading to the other nodes are arrows in both the left and right directions.

After entering the Courses node, the user is led in the courses option, where a list of available courses is given. From this point, it is possible to send email, or to use a calendar. For better user orientation there is a site map of the system. From this map a direct access to any of the other nodes is possible as well. If the user chooses calendar, it is possible to verify any chosen date after entering it in the required fields. Sending an e-mail is possible through choosing the email option.

After choosing any of the courses offered, the user is in the classroom. The case for Digital Image Processing classroom is shown on Figure 3. From here are available the course info, schedule of the course, announcements made by the teacher, help room, the content and students home pages. In the classroom is the connection between second and third levels, as well. After choosing the "lessons" button, the access to the content and course material is opened on a separate level.

November 6 - 9, 2002, Boston, MA 
The organizing of the content is in a house, separated in rooms, every room has walls and every wall has bricks. Here, the house corresponds to a book, room to a chapter, wall to a paragraph and brick to a basic concept [1].

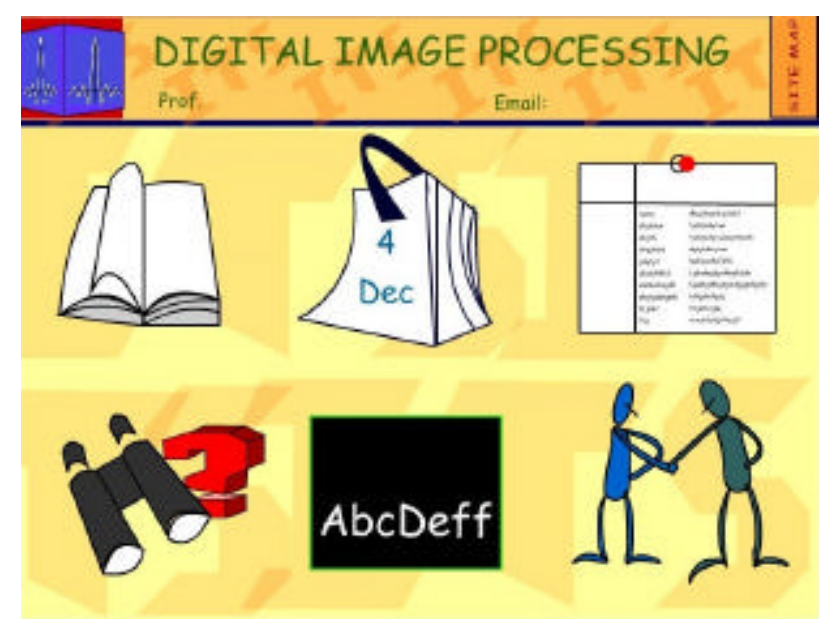

FIGURE. 3

Digital IMAGE PROCESSING ClASSROOM

There are rigorous and intuitive definitions, qualitative and quantitative examples for everyone of the bricks. The text is supported with audio. And after finishing the presentation of the information in the brick, there is a possibility for passing through it once again. All the bricks in one wall are linked, so that after seeing the first brick, the user is able to go to the following and so on to the end of the wall. Passing through the walls and the recommended time for spending on a brick are specified in the Schedule of the course. In case the maximum recommended time for a brick is over the system automatically loads the consecutive one.

Links to lab session and an exercise are available for each brick. They are also performed online through connection to a MATLAB server.

The Support Center is directly accessible from Main Interactive Hall. As mentioned before, it is designed to be on the fourth level. From the Support Center are accessible Digital Library, Help Room, Chat Room, and Students Home pages. From here, it is possible to send email or use a calendar as in the Courses option.

Help and Chat Rooms are still in process of developing. They are really meaningful when the system is entirely independent on WWW, from the normal class environment.

\section{Preloader for GUI}

Due to the way they are written, Flash files are streaming file formats [6]. The term streaming describes the way in which data flows from the HTTP server to the end user's computer. Normally, before a browser can render a graphic, text or other element to the screen, the entire file must be downloaded from the Web. Thus, if a slow connection exists somewhere between the end user and the server, content loads very slowly because the file has to be fully downloaded before it can be viewed. Flash files, however are written sequentially; that is, Frame 1 is written, then Frame 2, and so on. Therefore, as soon as the data associated with Frame 1 is downloaded, it can be played even if the remainder of the file is not downloaded.

Streaming multimedia files over the Web will only play smoothly if the rate of playback is less than or equal to the rate of downloading. Any time the rate of playback is faster than the rate of download, a noticeable pause may occur because the multimedia asset must stop and wait for more data to load.

Flash movies always stream. The effectiveness of SWF streaming ultimately depends on the amount of data required for each frame. If large bitmaps or lengthy sounds are present, the data requirements for the frame become quite large and can negatively affect the streaming process. Although optimizing may yield some decrease in file size, often it is not enough to avoid a presentation pause.

The size of present Flash movie is $1.45 \mathrm{MB}$, which presents a need of using preloader.

Simply, it is a set of frames that loops while waiting for the content to load. Once a particular frame is loaded it jumps to another particular one while all the rest is loaded. Preloader is only detected when the connection requirements are slow.

\section{ONLINE LAB-SESSIONS THROUGH MATLAB WEB SERVER}

MATLAB Web Server (MWS) exploits the power of WWW by integrating the visualization/computational capabilities of MATLAB with the remote access capabilities of WWW browsers. It enables creating MATLAB applications in such a way that allows students to transfer their data through WWW to MATLAB for the necessary computations. Consequently, the output of the computation, whether numerical or graphical, is accessible on the client's browser through the WWW [4].

These features of MWS allow students to run MATLAB applications through the Internet without having MATLAB software installed on their machines. For achieving this, a Web browser runs on the student's PC, whereas MATLAB, MWS and the Web Server daemon (httpd) run on the server machine.

The interaction between students and server is illustrated on Figure 4. As shown, the students are interacting with MATLAB over a network using a TCP/IP protocol. This interaction takes place through HTML forms, which serve as a point-and-click GUI for MATLAB applications. These capabilities not only allow students to use MATLAB based tools without any prior MATLAB programming knowledge, but also prevent unauthorized student access to source code and the MATLAB command line.

November 6 - 9, 2002, Boston, MA 


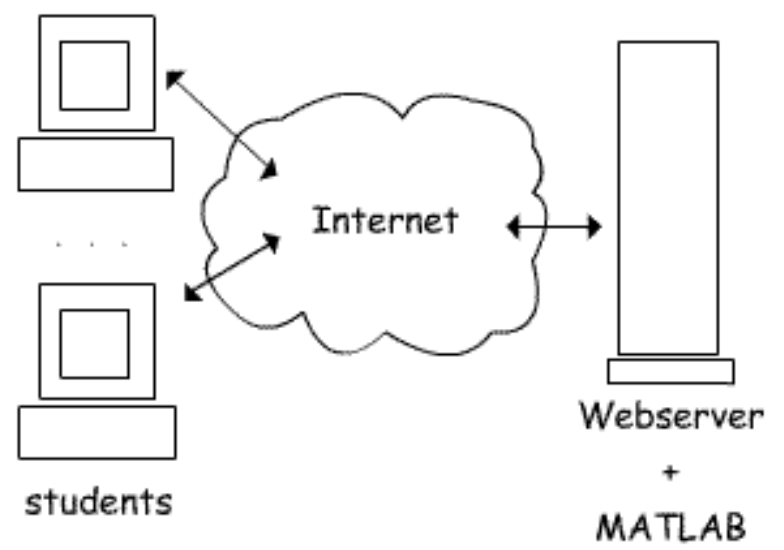

FIGURE. 4

StUdENT/SERVER STRUCTURE BASED ON MATLAB WEB SERVER

The interaction between students and MWS and the role of HTML forms as GUIs for MATLAB application is shown on Figure 5.

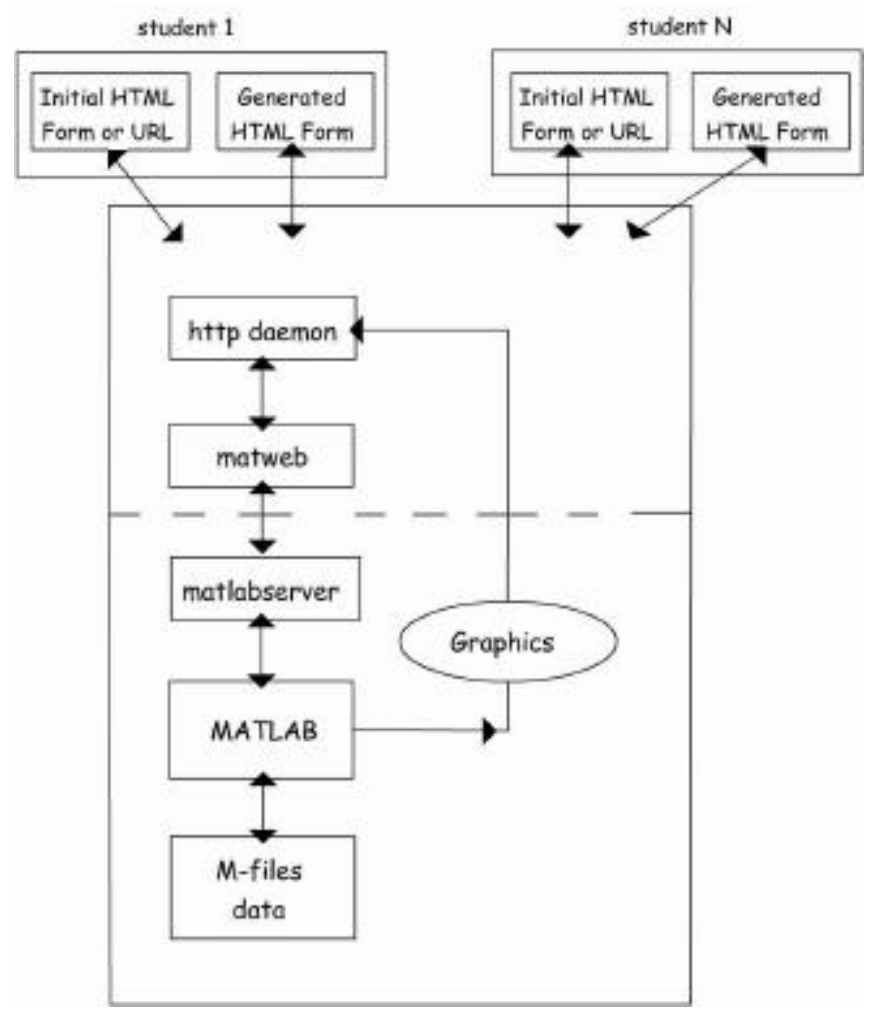

FIGURE. 5

CONFIGURATION AND INTERACTION BETWEEN STUDENTS AND MWS

The student enters appropriate data in the relevant spaces before pressing "Generate filter" in an HTML form (Figure $6)$.

The data is then transferred to the server machine, housing the MATLAB software and the corresponding mfile. This step is initiated by matweb (the client of MWS that uses
Common Gateway Interface (CGI)), which takes the data from the HTML form and transfers the information to MWS. The MWS then runs the application that is written in m-files to produce desired responses.

These responses travel in the opposite path to arrive at the related output HTML file. This sequence of information transfer is depicted on Figure 7.

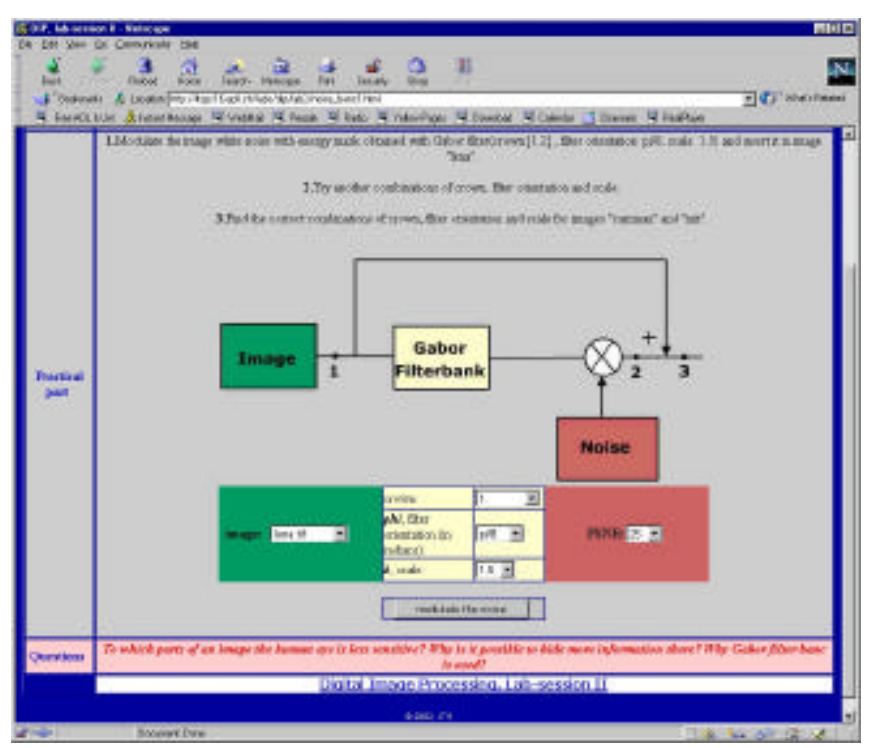

FIGURE. 6

AN EXAMPLE FOR INPUT HTML FORM

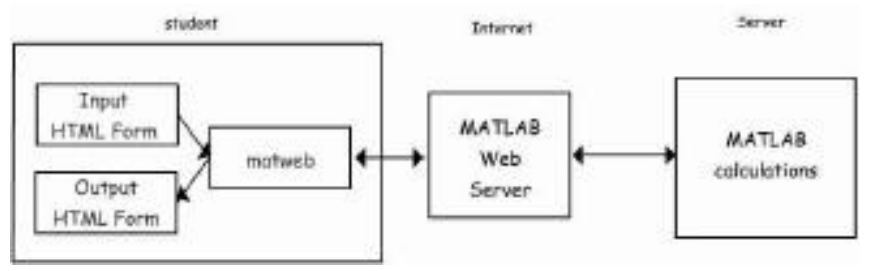

FIGURE. 7

AN INFORMATION FLOW GRAPH BETWEEN STUDENTS AND MWS

The example provided is about image watermarking. As it is shown on the input HTML form, the image under study is first filtered using Gabor filter bank, and the output is used for modulating a white noise which represents the watermark and is added to the original image. The student chooses the input image, filter characteristics (phase, orientation and scale) and PSNR as shown on Figure 6. These variables are sent to MATLAB and the result of the calculations is displayed in the output HTML form on Figure 8.

In the output form the input image, the modulated image noise and the output image are displayed. The input image with added uniform white noise is displayed as well, so that it is possible to be compeared with the watermarked image.

November 6 - 9, 2002, Boston, MA 


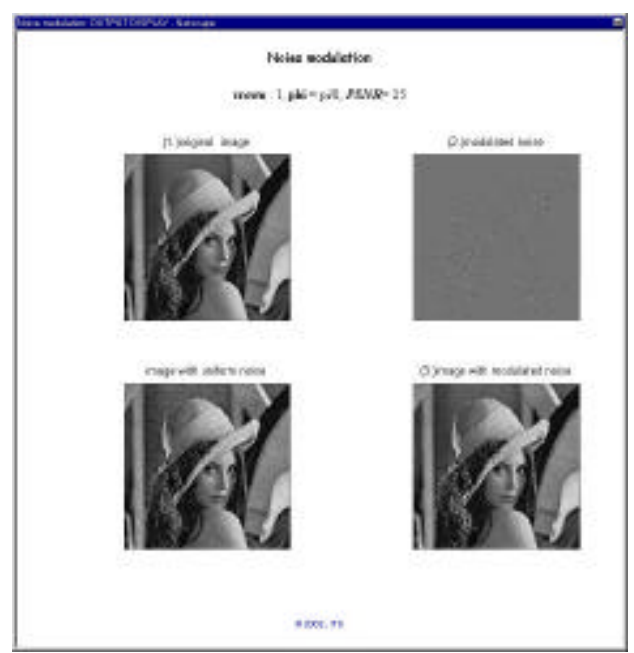

FIGURE. 8

EXAMPLE OF AN OUTPUT HTML FORM

\section{COMPLEMENTARY TOOLS: DIGITAL LIBRARY AND CALENDAR}

In order to make more recent information, supporting the course content, available to the students, a tool like a Digital Library is created. It consists of links with a database, where some publications or relevant optional material is stored. The interface of the Digital Library is depicted on Figure 9.

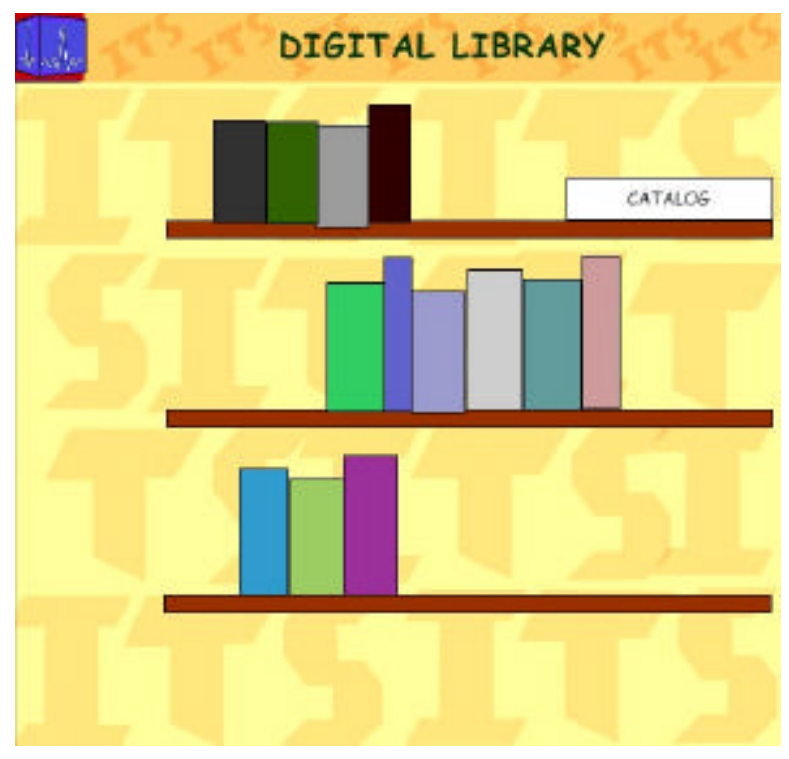

FIGURE. 9

DIGITAL LIBRARY

For better organizing of the students' time a calendar is offered. It gives the possibility for the user to check each of the dates he/she is interested in providing some information concerning the schedule of the course the user is subscribed in. The calendar's interface is depicted on Figure 10.

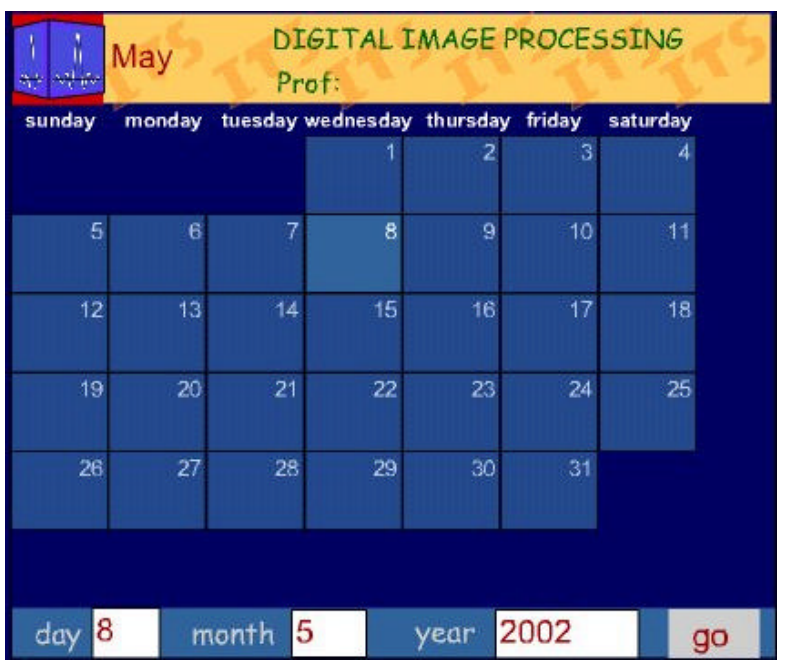

FIGURE. 10

CALENDAR/SCHEDUle

\section{CONCLUSIONS AND FUTURE WORK}

The virtual classroom for multimedia teaching on WWW in Digital Signal and Image Processing described here, is meant to be available independently, but while tested, it is as a support to a normal class.

There are many benefits to learning in a virtual classroom setting, which are independent of the material covered in the course. Mainly, the students get the ability to get away from their other responsibilities and concentrate on learning the course materials greatly increases the amount that they learn and retain. Using MATLAB Web Server makes more efficient understanding the particular problem addressed in each exercise. Using it, the student will be concentrated on technique applied on a signal or an image, playing with some parameters concerning the problem treated and viewing the result in a graphical or numerical representation. An independent tool for testing is going to be developed, so that the course exams will be performed on-line. This will allow the courses to be held entirely and independently online.

\section{REFERENCES}

[1] Bogdanova, I, Khan, R, M, Kunt, "Multimdia teaching on WWW: a new approach",proceedings of Networked Learning 2002, Berlin

[2] Kerman, P, "Macromedia Flash 5 in 24 hours", SAMS, Indiana USA, 2001

[3] Kunt, M, "Digital Signal Processing", Artech House, 1986

[4] MATLAB Web Server manual, Mathworks, Inc., 1998

[5] Musciano, C, Kennedy, B, "HTML, the definitive Guide", O'REILLY, 1998

[6] Mohler, J, L, "Graphics, Animation \& Interactivity with Flash 4.0", Delmar Thomson Learning, 2000

November 6 - 9, 2002, Boston, MA 